\title{
Special issue on Similarity, Correlation and Association Measures
}

\section{Dedicated to the memory of Lotfi Zadeh}

\author{
Ildar Batyrshin ${ }^{\mathrm{a}, *}$, Valerie Cross ${ }^{\mathrm{b}}$, Vladik Kreinovich ${ }^{\mathrm{c}}$ and Maria Rifqi ${ }^{\mathrm{d}}$ \\ ${ }^{a}$ CIC, Instituto Politécnico Nacional, México \\ ${ }^{\mathrm{b}}$ Computer Science and Software Engineering, Miami University, Oxford, OH, USA \\ ${ }^{\mathrm{c}}$ University of Texas at El Paso, USA \\ ${ }^{\mathrm{d}}$ LEMMA, University Panthéon-Assas - Paris, France
}

Lotfi Zadeh, the father of fuzzy logic, opened doors to a new world of science where "everything is a matter of degree" be it set membership, logical truth, similarity, preference, etc. Fuzzy logic paved the way to new directions for research in mathematics, artificial intelligence, control, modeling of uncertainty, decision making, computational intelligence, soft computing and hybrid intelligent systems.

Some of these research directions are based on the concept of a fuzzy relation introduced and studied by Zadeh in his papers. The concepts of max-min composition, max-min transitivity and fuzzy similarity relation proposed by Zadeh as generalizations of the corresponding crisp concepts play important roles in hierarchical clustering analysis based on similarity measures, in decision making and, more generally, in fuzzy mathematics. In recent years, there has been an increasing number of papers establishing relationships between fuzzy logic (in the wide sense) and similarity, correlation and association measures. Such measures have been introduced for fuzzy sets of different types: fuzzy, intuitionistic, rough, soft sets etc. Some concepts and approaches used in the analysis of fuzzy relations and, generally, in fuzzy logic are also used in the analysis and construction of similarity and association measures.

Similarity, correlation and association measures play important roles in data analysis and data mining, in data classification, pattern recognition and

\footnotetext{
${ }^{*}$ Corresponding author. Ildar Batyrshin. E-mail: batyr1@ gmail.
}

machine learning. With the development of new methods of data mining and machine learning, the number of data analysis problems that can be solved by these methods have increased. The areas of application of these methods have also expanded, and nowadays there exists a large number of similarity, correlation or association measures for different domains.

The Guest Editors of the Special Issue on "Similarity, Correlation and Association Measures" tried their best to present in one volume different approaches to definition, construction and application of similarity, correlation and association measures for various domains, types of data and application tasks. The paper "Towards a general theory of similarity and association measures" shows that the concept of fuzzy relation introduced by Zadeh can serve as a basis for joint analysis of similarity and association measures on a universal domain, and many results obtained for fuzzy relations can be extended on these measures. Other papers contain surveys and research results on similarity, dissimilarity and association measures for $2 \times 2$ tables, ontologies, soft matrices, multisets, intuitionistic fuzzy information, images, etc., and their applications.

We hope that this special issue will be helpful for establishing relationships between measures defined for different data types, for transferring the measures and their properties from one domain to another one, and for possible generalizations of existing measures. We think that such generalizations can be based on the 


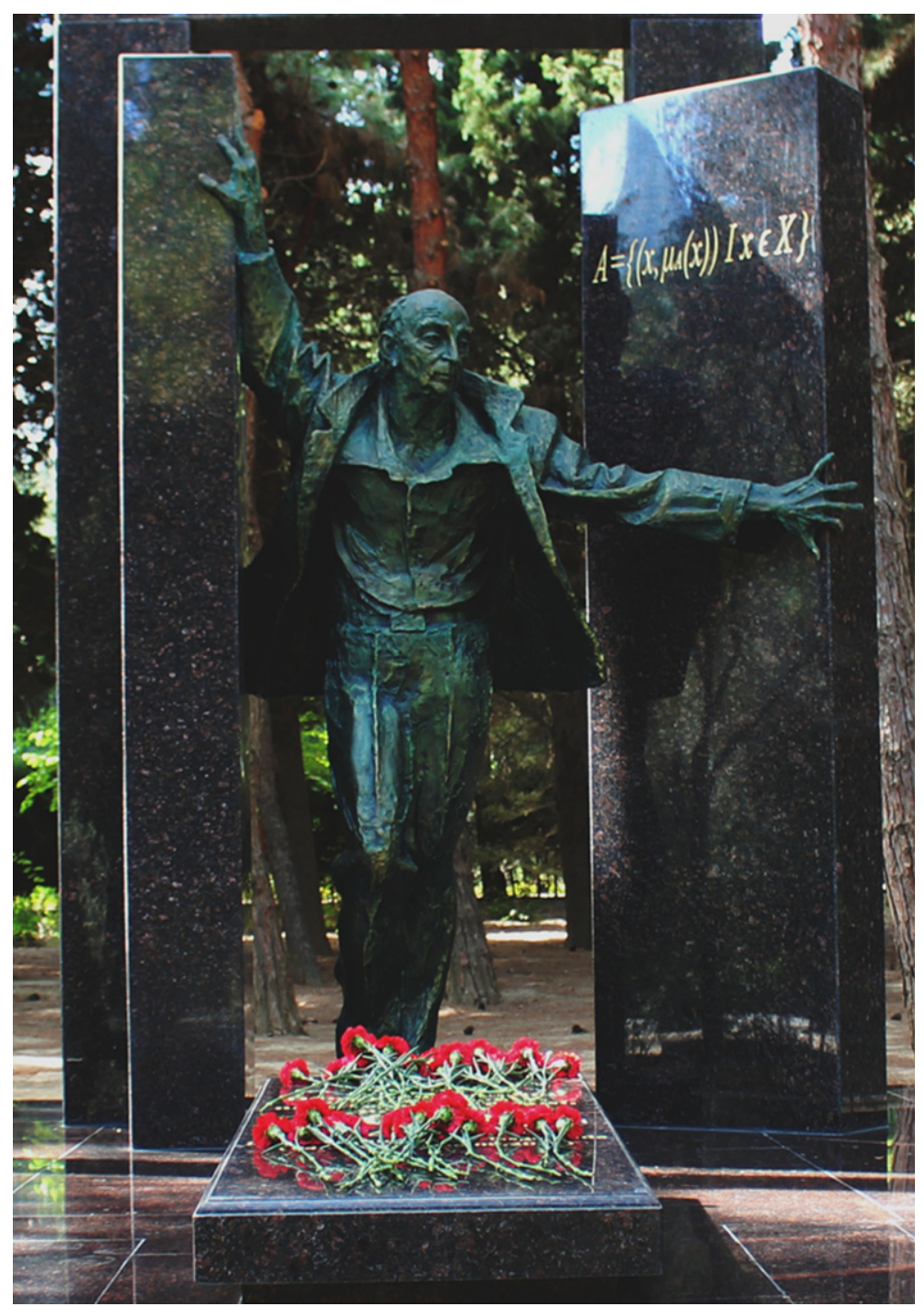

Monument of Lotfi Zadeh on his grave on Alley of Honor, Baku, Azerbaijan.

mathematical models and methodology developed in the theory of fuzzy relations and, more generally, in fuzzy logic.

Guest Editors of this Special Issue thank Dr. Reza Langari, the Editor-in-Chief of the Journal of Intelli- gent and Fuzzy Systems, and Mr. Maarten Fröhlich, Publisher of this journal, for their support in publishing this Special Issue. Special thanks to Dr. Shahnaz Shahbazova for providing us the photo of the monument erected on the grave of Lotfi Zadeh. 